BARRAGES-VOUTES

\title{
I. -- Evidences, paradoxes et contradictions dans leur conception et leur calcul
}

\author{
ARCH DAMS \\ I. -- Evident facts, paradoxes \\ and contradictions in their design
}

PAR

P. PATIN, DIREcteUr gÉNḱral DE LA C.I.T.E. (*)

\begin{abstract}
Compte tenu de l'augmentation du taux de travail du beton dans les barrages-voutes, le problème de leur appui sur le rocher de fondation devient insoluble avec les techniques classiques. D'autre part, l'encastrement difficile à réaliser provoque la fissuration du rocher et du barrage et, tout en exigeant plus de matière, diminue la sécurité.

Il semble recommandable d'évoluer vers des ouvrages utilisant mieux la double courbure et s'appuyant, par l'intermédiaire d'une articulation, sur un élément de répartition des contraintes et de régularisation de la ligne d'appui.
\end{abstract}

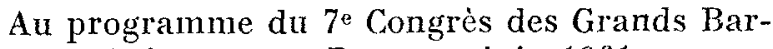
rages qui s'est tenu à Rome en juin 1961, aucune question ne portait à proprement parler sur les barrages-voûtes.

${ }^{\star}$ ) C.I.T.E. : Compagnie d’Ingénieurs et Techniciens d'Etudes, 8, place Vendôme, Paris $\left(1^{\text {er }}\right)$.
ET

\author{
G. DEGEORGES, \\ INGSENEUR CONSEIL A TAA C.I.T.E. $\left({ }^{*}\right)$
}

\begin{abstract}
As a resnlt of the increasingly higher worling stresses affecting the concrete of modern arch dams, the problem of their abutment against the fonndation rock can no tonger be solved by conventional methods.

The restraining of the dam ends ...... almatls a difficult problem - causes cracks to form in both rock and structure, in addition to requiring greater quantities of malerial and leaving a smaller safety margin.

The design trend to be recommended would appear to the tousards structures making betler use of the double curvature principle and being supported through a hinge on a bearing block giving a more satisfactory line of load application.
\end{abstract}

Cependant, sous l'influence, d'une part, d'une catastrophe récente présente à tous les esprits, d'autre part, des réalisations effectuces par les ingénieurs italiens et portant toutes la marque d'une conception originale, il est bien certain que les barrages-voûtes ont été largement présents dans les préoccupations des membres du Congrès. 
Des communications sur les barrages pour vallées larges, des échanges de vues faits à cette occasion, des articles et des rapports des congressistes les plus éminents, des conversations au cours des visites des grands ouvrages et des enseignements recueillis de ces mêmes visites, il semble résulter, chez les ingénieurs qui s'intéressent particulièrement aux barrages-voutes, une sorte de malaise. Des problèmes se posent, des conceptions s'affrontent, des notions que l'on croyait acquises redeviennent hypothétiques.

Le présent article n'a pas pour but de résoudre ces problèmes, mais plutôt d'essayer, dans l'incertitude où se trouvent actuellement les projeteurs, de discerner les causes de leurs difficultés, et d'esquisser les grandes lignes d'une tendance nouvelle.

\section{LE ROCHER}

Un premier point, qui transparaît nettement dans un assez grand nombre de communications (citons par exemple, indépendamment du rapport général de MM. Manuel Rocha et Laginha Serafim, ceux du Docteur Huggenberger, de M. Semenza, du Groupe de Travail français sous la direction de M. Duffaut) est le souci du comportement du rocher en tant qu'appui du barrage.

Il est bien certain que l'on sait faire maintenant des bétons travaillant d'une façon très régulière à des taux élevés, et il est non moins certain que chaque fois que l'on essaie à la presse un morceau de roche, ou lorsqu'on mesure sur un échantillon le module d'élasticité dynamique, on obtient des résultats qui permettent presque toujours de dire que le rocher a des caractéristiques comparables à celles du héton, sinon meilleures.

Ceci malheureusement n'est valable que pour un élément de faible dimension; mais, alors que l'on sait couler de volumineux plots d'un béton sensiblement homogène et presque isotrope, le rocher, par contre, pris en place, présente toutes sortes de défauts, auxquels malheureusement ne remédient pas complètement les différents traitements qu'on peut lui faire subir. Les injections les mienx faites ne réussissent pas - l'expérience l'a prouvé — à faire d'un rocher diaclasé un bloc homogène et il reste, après la construction des ouvrages, dans toutes les roches, des plans de séparation plus ou moins bien collés qui obligent l'ingénieur à traiter les appuis d'une façon tout à fait différente des ouvrages proprement dits.

De nombreuses études ont été faites sur le contact béton-rocher, sur les efforts de cisaille- ment que ce contact est susceptible de transmettre; mais, à l'intérieur du rocher lui-même, nous sommes bien forcés d'avouer que nos connaissances sont encore rudimentaires el que le comportement effectif de la matière dépend d'un nombre considérable de facteurs sur lesquels nous agissons peu ou difficilement :

-- nombre et direction des plans de clivage;

-- coefficient de frottement dans ces différents plans;

- action de collage plus ou moins importante des différents types d'injection;

- influence de l'eau d'infiltration et des éléments qu'elle transporte;

- influence de la pression et de ses variations;

- séquelles des traumatismes subis par le rocher pendant et après la construction, etc.

C'est pourquoi, nous sommes tentés, pour pexturber le moins possible le terrain dans son état naturel, de lui transmettre des contraintes faibles et bien réparties. C'est sans doute à une de ces préoccupations que répond le «pulvino».

On constate cependant, et c'est là un des premiers paradoxes sur lesquels nous aimerions attirer l'attention, que les habitudes conduisent à utiliser le terrain d'une façon peu rationnelle; en effet presque toujours le fond des vallées, constitué par des rochers dont l'érosion est relativement récente, présente une qualité, une homogénéité et très souvent une compacité qui permettraient de lui transmettre des efforts importants sous des angles relativement faibles. Au contraire, les parties moyennes et hautes des vallées sont presque toujours météorisées, très diaclasées, souvent feuilletées par des plans verticaux parallèles à l'axe de la vallée et par conséquent constituent des appuis plutôt mauvais.

Or, la plupart des ouvrages sont concus de façon à apporter au fond de la vallée un effort presque vertical que les théories actuelles conduisent d'ailleurs à prévoir assez faible dans le cas des barrages-voûtes, alors qu'au contraire les arcs moyens et supérieurs de ces barrages viennent prendre appui sur les versants avec des angles très aigus, de l'ordre de 20 à $30^{\circ}$, tout en leur apportant des efforts considérables.

\section{Contraintes dans le rocher}

D’une façon générale, il nous paraît dangereux de transmettre au rocher, directement, des contraintes très élevées (c'est-à-dire celles que l'on admet dans un bon béton), et ceci que le rocher soit ou non fortement diaclasé (ou autrement dit, que la distance moyenne des diaclases soit de l'ordre du décimètre ou du mètre).

Il y a à ceci deux raisons essentielles :

$1^{\circ}$ Il est presque improbable que, dans la 
famille des plans tangents aux deux parements, le long de la fondation, il n'y ait pas une zone dans laquelle ces plans coïncident avec l'une des trois directions de plans de diaclases qui existent dans presque tous les rochers. Dans cette zone, les contraintes, loin de se répartir en s'étalant dans un massif homogène et isotrope conforme aux idées de Boussinesq, vont se transmettre en profondeur, restant bien sagement cloisonnées entre les plans tangents aux deux parements, matérialisés par des diaclases. On aura donc un point faible de la fondation, dû, non pas à la qualité du rocher qui peut être aussi bon qu'ailleurs, mais au tassement élastique d'une masse indéfinie sous une contrainte restant constante en profondeur. Les cisaillements dus à des variations de compression brutales vont séparer le rocher en tranches plus ou moins parallèles à la direction générale des forces.

Pour peu que les efforts soient inclinés, c'est-àdire comportent une part importante de cisaillement, les plans de diaclases vont s'ouvrir, introduisant la pression de la retenue dans le rocher à la base de l'ouvrage.

$2^{\circ}$ On objectera aisément qu'une grande part des efforts sera transmise par frottement. Mais notre expérience personnelle dans des domaines divers, confirmée par celle de spécialistes de la mécanique des roches (M. Biarez en particulier), montre que, loin d'être constant, comme l'indiquerait la loi de Coulomb, le coefficient de frottement diminue quand la pression augmente; ceci est valable aussi bien pour les rochers que pour les métaux et pour bien d'autres matériaux. Ne comptons done pas trop sur le frottement pour transmettre des efforts élevés, dès que la continuité du rocher, ou du béton, n'est plus assurée.

On peut également objecter que les diaclases sont généralement colmatées par les coulis d'injection de «consolidation». Colmatées sans doute, mais collées, c'est peu probable. De toutes façons l'expérience prouve également que la résistance à la traction et au cisaillement des coulis d'injection est négligeable.

\section{Application des efforts}

Les grands ouvrages-voùtes sont très lourds et représentent souvent 70 à $90 \%$ du poids des barrages-poids qu'ils remplacent. Il paraît irrationnel de ne pas utiliser cet élément pour transporter les efforts vers les parties saines du rocher.

La logique voudrait donc qu'une part plus élevée de la poussée de l'eau soit transmise au fond et aux parties les plus profondes des flancs de la vallée, en utilisant au besoin des angles d'attaque plus faibles que ceux classiquement utilisés, mais en s'efforçant de limiter le plus possible les cisaillements, l'idéal étant évidemment de n'apporter au rocher que des compressions pures relativement faibles.

Ceci conduirait à des ouvrages ayant de forls surplombs vers l'amont et nous savons que cette condition tend à résoudre un autre des problèmes que se posent souvent les projeteurs, celui des tractions dans les consoles à l'amont des ouvrages.

\section{LE CALCUL}

Parlons done un peu de ces fameuses consoles. Depuis la mise au point de la Trial Load Method, on ne parle plus, pour le calcul des grands barrages-voûtes, que d'arcs ef de consoles. Cette conception a dérivé, petil à petil, comme nous le reverrons plus loin, des idées de Resal ef de Stucky qui, dans l'ignorance où nous sommes de la façon dont travaillent les harrages-voûtes, avaient cherché à les décomposer en éléments horizontaux et verticaux; mais nous sommes bien obligés de dire que nous ne savons pas en réalité comment travaille la matière dans les barrages-voûtes.

Nous touchons là à un mode très dangereux d'évolution de la pensée. La réflexion suggère l'essai d'un mode de calcul sans le justifier entićrement. Mais, la possibilité du calcul étant établie (et c'est peut-être un malheur que les calculs les plus complexes soient devenus faciles..), le projeteur qui l'effectue, trouvant des résultats cohérents, les prend pour argent comptant et finit par croire à la réalité des hypothèses. Malbeureusement le fait qu'un calcul puisse être mené à son terme ne prouve absolument rien quant à la valeur de ces hypothèses de départ.

Le seul moyen d'approcher la réalité est l'ex. périmentation sur modèle-réduit - et encore à condition de faire intervenir le poids de l'ouvrage - et celte expérimentation nous permet seulement de déterminer l'allure générale des isostaliques et l'ordre de grandeur du taux de travail.

Apres construction de l'ouvrage, on peut enregistrer les contraintes en place, si l'on a pris soin de placer, comme on le fait le plus souvent aujourd'hui, des témoins fidèles dans le béton du harrage. Or, lorsque l'on compare les résultats du calcul effectué par la Trial Lond à ceux des essais sur modele et aux enregistrements dans l'ouvrage on est bien ohligé de constater des différences.

Dire que l'on est satisfait lorsque l'écart ne 
dépasse pas $20 \%$ revient simplement à dire que l'on ne sait pas si le mode de calcul est bon. Une autre méthode donnerait peut-être également 10 ou $20 \%$ d'écart, mais dans un autre sens et cette méthode n'en serait pas meilleure pour cela. La différence entre les deux atteindrait pourtant $50 \%$, mais, horrible détail, ce sont des différences de cet ordre de grandeur, nous voulons dire de $50 \%$, que l'on trouve quelquefois entre les résultats du calcul et ceux de l'étude sur modèle, et l'on se reportera avec intérêt au rapport de MM. Schultz, Scrivner et Copen, relatif à l'étude d'un projet fictif pour le barrage de Friant. Encore s'agit-il d'un ouvrage dans lequel la vallée étant large et les consoles courtes, ces dernières peuvent peut-ètre jouer effectivement ce rôle de consoles qu'on leur prête.

Mais que dire des ouvrages minces de grande hauteur, dans lesquels la coupe en clé fait irrésistiblement penser à une lame de sabre (citons Vajon, Le Gage, Tolla et bien d'autres). Coniment peut-on parler de consoles et d'encastrements dans de tels ouvrages?

Un autre point important dans le calcul du barrage est la répartition des contraintes dues à l'appui sur le rocher. Lorsqu'on fait un calcul par arcs et consoles, on suppose implicitement que les déformations du rocher sont nulles, ou dans certains cas qu'elles sont simplement proportionnelles aux contraintes, en tablant sur des coeflicients d'élasticité que l'on peut à la rigueur faire varier d'une façon plus on moins continue le long de la fondation. En fait, on admet toujours que cette fondation se déforme très peu, sans quoi on ne pourrait parler d'encastrement.

Or, il est hien certain que le barrage ne s'appuie que sur ce qui résiste et il se produit toujours de fortes concentrations de contraintes sur les points durs, que ceux-ci soient créés par une variation dans la forme de la fondation ou par une zone de rocher plus résistante que les zones voisines. De tels points ne sont pas exceptionnels dans les appuis de la plupart des barrages et on peut se demander ce que devient dans ce cas le calcul que l'on a pu effectuer.

Inversement, comme nous l'avons montré plus haut, des points faibles «apparents» peuvent exister dans une fondation de rocher relativement homogène mais finement diaclasé.

Enfin certaines roches ont un coefficient d'élasticité très bas et se déforment considérablement sous les forces élevées qui leur sont appliquées.

Là se justifie une second fois l'apparition du «pulvino» dont le rôle serait de régulariser la ligne de fondation et aussi de permettre une répartition de contraintes suffisamment faibles pour: que l'on puisse admettre que l'ensemble du rocher résiste d'une façon presque homogène.
Revenons-en à l'encastrement.

Lorsqu'un ingénieur construit une charpente en béton et qu'il veut encastrer une poutre dans un pilier, il prend grand soin de réaliser un ferraillage qui lie d'une facon parfaite la poutre au pilier. Il tient plus grand compte des dimensions relatives des deux pièces et il veille à ce qu'en aucun cas il ne puisse se produire de fissures remplaçant l'encastrement par une articulation. Or, dans le cas de grands barrages, des « consoles» dépassant une centaine de mètres de hauteur sont «encastrées» dans le bon rocher de quelques mètres, sans aucun ferraillage, ni dans le rocher bien entendu, ni dans le béton et, en outre, les joints de reprise horizontaux constituent les amorces de fissuration idéales pour qu'il ne se produise aucune espèce d'encastrement. Même si le béton ne se fissure pas (ce que l'on constate le plus généralement en s'en félicitant) le rocher a bien des chances de le faire : il est fissuré d'avance. Or, pour qu’il y ait véritable encastrement, il faut bien que le rocher à l'amont de la fondation travaille à la traction. Est-ce possible?

\section{L'HYPERSTATISME}

D'ailleurs, il faut bien le dire, et M. Manuel Rocha l'a écrit en toutes lettres, que cet encastrement est la bête noire des projeteurs. En effet, il introduit des tractions qui, évidemment, sont des contraintes parasites, tendant à provoquer la fissuration du béton et qu'il faut, en outre, compenser par une valeur équivalènte de compression; et ceci nous conduit à nous poser le problème de l'byperstatisme...

Si l'on néglige momentanément les contraintes internes dans le béton, qu'elles soient dues au retrait, à la température ou à d'autres facteurs, le problème à résoudre par le barrage est de transmettre au terrain la résultante de deux forces, à savoir : la poussée de l'eau et le poids du barrage.

C'est une évidence de dire que l'idéal serait de ne transmettre au terrain que des compressions, et cet idéal ne peut être atteint que par des ouvrages isostatiques.

Le fait d'encastrer les appuis des barrages les rend hyperstatiques et introduit dans les zones d'appui des moments de flexion qui sont bien embarrassants. En effet, comme nous l'avons déjà dit, qui dit moment dit traction et il faut bien compenser ces tractions, qui n'existaient pas au départ et qui ne proviennent que de l'ouvrage lui-même, par un total de compressions équivalentes (ceci vu d'un point de vue purement stati- 
que, indépendamment de tous les ennuis que peuvent procurer. Ies fissures dues à ces tractions; et encore les sous-pressions qui s'introduisent par les fissures modifient-elles, et souvent dans le mauvais sens, l'équilibre statique de l'ensemble).

La figure 1 montre quelle est la répartition des contraintes dans une section d'encastrement et fait ressortir l'économie considérable de matière qu'on réaliserait si on pouvait travailler en compression hydrostatique.

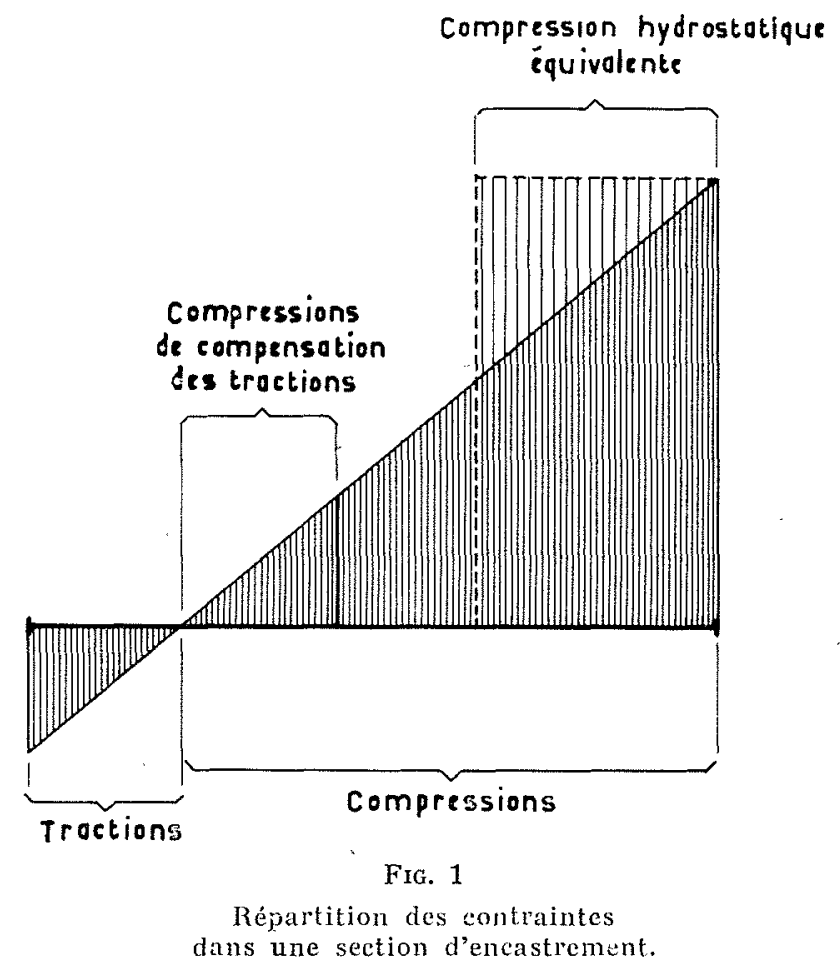

Et quand on $\mathrm{y}$ réfléchit, mieux on utilise le béton, plus ces moments deviennent obligatoirement importants.

Prenons par exemple un arc de $100 \mathrm{~m}$ de longueur, travaillant à $80 \mathrm{~kg} / \mathrm{cm}^{2}$ avec un module d'élasticité permanent de $240000 \mathrm{~kg} / \mathrm{cm}^{2}$. Son raccourcissement sous charge serait donc de $80 / 240000$, soit $1 / 3000$, ou en valeur absolue 'de $100 \mathrm{~m} / 3000=33 \mathrm{~mm}$.

Si cet arc pouvait se déplacer en glissant radialement sur ses appuis il se rapprocherail de $16 \mathrm{~mm}$ de son centre. Si l'on fait travailler le béton uniformément à $120 \mathrm{~kg} / \mathrm{cm}^{2}$, les chiffres deviennent $50 \mathrm{~mm}$ de raccourcissement et $25 \mathrm{~mm}$ de déplacement. La figure $2 a$ montre, très schématiquement, ce que devrait être le déplacement de l'arce, et la figure $2 b$ ce qu'est, en réalité, sa déformation ou plus exactement, ce qu'elle serait si l'arc était indépendant. Tout ceci sans tenir compte des déformations des appuis.

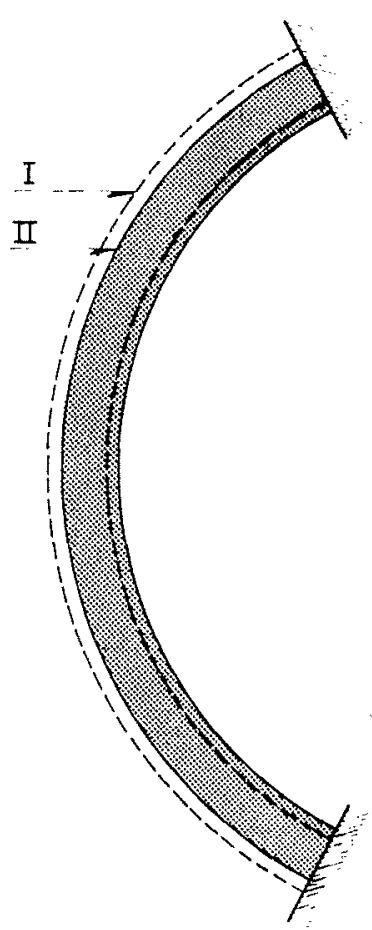

Ftg. $2 a$

Délormation d'un are sur appuis libres.

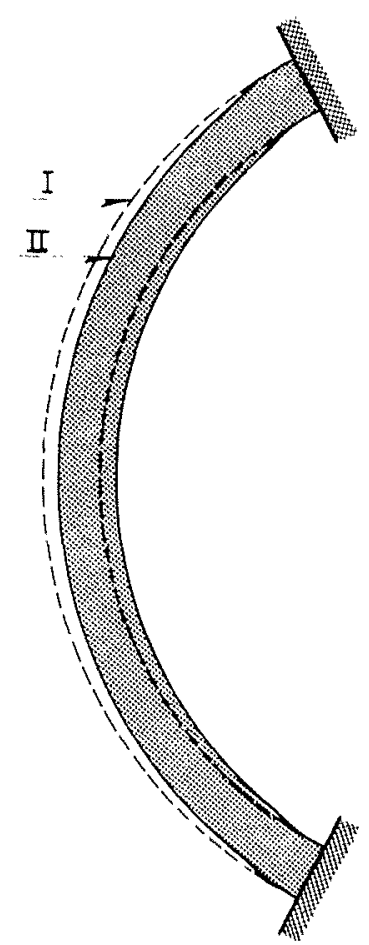

Fici. $2 b$

Deformation d'un are cncastré.
On constate que l'hyperstatisme, loin d'apporter un supplément de rósistance et de sécurité, n'est qu'une cause de torture des ouvrages, torture de plus en plus pénible au fur et à mesure que l'on utilise mieux la matière.

En créant le joint périmétral, les ingénieurs italiens ont sans doute cherché à lever aussi cette hypothèque. II ne semble pas cependant qu'ils soient allés au hout de l'idée, puisqu'après la construction ce joint est bloqué et ne permet ni articulation, ni glissement.

La tendance actuelle, pour utiliser le béton avec des taux de travail élevés, pour tenír compte de la rigidité et de la meilleure répartition des contraintes données par la double courbure, est de transformer le barrage-voûte simple en coupole mince, mais la coupole que l'on calcule comme une membrane est incompatible avec les moments élevés dus aux encastrements.

\section{LES OUVRAGES MINCES}

Lorsque l'on étudie la répartition des contraintes dans un barrage mince à double courbure, considéré comme une membrane, on admet qu'il n'existe aucun cisaillement transversal of la 
figure 3 que nous empruntons au live de M. Bourgin montre que l'on essaie de répartir selon deux directions, que l'on choisit généralement perpendiculaires, la résultante des forces de surface et de volume; autrement dit qu'il existe une surface moyenne que l'on considère comme surface funiculaire et que l'on décompose selon les axes en funiculaires linéaires. II est asse $z$ logique dans la plupart des barrages de choisir comme direction des axes la direction verticale et la direction horizontale, dans la mesure où le barrage est construit par plots séparés par des surfaces verticales. Mais si les

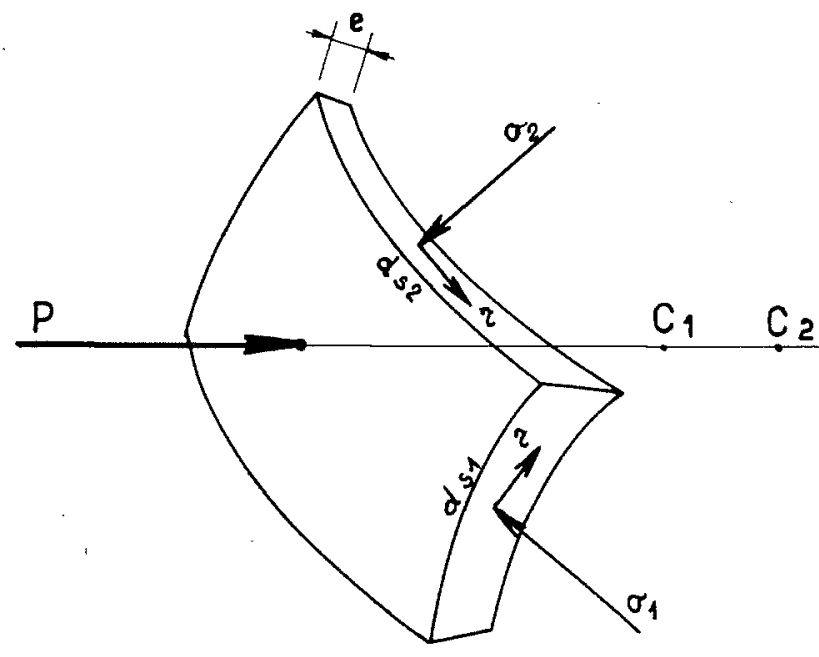

FiG. 3

Equilibre d'un élément de membrane.

éléments horizontaux (ou dans certains cas un peu inclinés sur l'aval) sont toujours des ares, les éléments verticaux ne sont en aucun cas des consoles.

En effet, ils ne transmettent que des efforts de compression perpendiculaires à leur section; par conséquent, on ne peut les considérer que comme des éléments d'arcs ou de voûtes tronqués, ne s'appuyant, d'une part, que sur la fondation et, d'autre part, que sur leur propre poids, à. moins que l'on puisse apporter à la partie haute de cette portion d'arc une force supplémentaire destince à encaisser une certaine réaction. Nous répétons que ce calcul n'est valable que s'il n'y a aucun cisaillement transversal et par conséquent s'il n'y a pas d'encastrement à la fondation de l'ouvrage. Dans ce cas, un ouvrage mince à double courbure se calculera assez facilement en le décomposant en arcs horizontaux et en demi-arcs verticaux à la condition expresse qu'ils ne soient pas encastrés, ce qui est d'ailleurs la condition sine qua non pour qu'on puisse le considérer comme une membrane dans le calcul.

Et ceci, très curieusement, nous conduit à faire la critique du mode de calcul actuel des barrages-voûtes et à faire se rejoindre, à travers les voûtes classiques, les harrages poids-voûtes et les coupoles.

Avant d'examiner en détail le calcul des ouvrages minces par la théorie de la membrane, il nous paraît nécessaire de revoir l'évolution des idées dans le domaine du calcul des barragesvoùtes.

\section{QUELQUES MOTS D'HISTORIQUE}

Pour résumer brièvement l'évolution de la technique en matière de construction de barrages-voûtes depuis le début du siècle, on peut dire que cette évolution a surtout porté sur deux points essentiels :

$1^{\circ}$ Augmentation progressive du rapport $c / h$ de la corde de l'arc situé en crête du barrage à la hauteur maximale de l'ouvrage au-dessus de sa fondation;

$2^{\circ}$ Accroissement sensible des contraintes théoriques maximales.

Cette évolution est sans doute la conséquence des progrès obtenus d'une manière générale dans l'étude des matériaux, tant en ce qui concerne leurs qualités que le contrôle de leur régularité, mais elle est aussi et surtout étroitement liée à la personnalité et à la hardiesse des grands ingénieurs qui ont attaché leur nom à ce domaine particulier du génie civil.

On peut en effet difficilement citer d'autres cxemples dans l'art de construire moderne où les progrès réalisés doivent aussi peu à la théorie pure.

Il est en général d'usage qu'après une découverte fondamentale dont la validité du principe est vérifiée, la technique progresse en s'appuyant pas à pas sur la recherche théorique. C'est alors souvent à la collaboration intime du constructeur et du théoricien que revient le mérite de l'amélioration logique et continue du procédé.

Il en va tout autrement pour les barragesvoûtes, puisque trois quarts de siècle après que les premiers grands théoriciens se soient penchés sur ce problème, il faut bien avouer que nous ne savons que très imparfaitement les calculer.

Les apports d'une expérience déjà longue sont également des plus modestes. Qu'il s'agisse des méthodes extensométriques ou des mesures topographiques, on connaît bien les difficultés d'interprétation auxquelles on se heurte, difficultés inhérentes en particulier au nombre de facteurs susceptibles d'influencer simultanément les déformations.

Beaucoup de barrages-voutes ont cependant été construits dans le monde et d'indiscutables progrès réalisés, qui doivent bien davantage à l'intuition, souvent à l'audace et parfois au génie 
de leurs autreurs qu'aux perfectionnements des procédés de calculs.

Déjà, avant la fin du siècle dernier, deux parmi les plus grands spécialistes français de la mécanique appliquée, Maurice Lévy et Jean Resal avaient abordé le problème des barragesvoûtes.

Resal, en particulier, s'il avait préconisé le calcul par arcs indépendants et émis le premier l'idée des «voutes actives», avait par contre, sur la foi d'un calcul sommaire d'ajustement de clé, condamné l'emploi de barrages-voûtes dans le cas où le rapport $c / h$ dépasserait la valeur 2,5 . Il avait, en effet, montré qu'au-delà de cette valeur, l'importance des contraintes de traction était telle que l'on devait normalement s'attendre à des ruptures.

Tous les ingénieurs qui ont eu l'occasion de procéder à des calculs de ce genre sur un exemple concret ont pu vérifier le bien-fondé apparent des résultats de Resal.

Ce dogme du rapport $c / h$, ainsi énoncé par un théoricien aussi éminent a, semble-t-il, longtemps mis un frein à l'évolution de la technique des barrages-voûtes. Il est vrai, toutefois, que la construction des barrages-vôttes n'a connu son véritable essor qu'avec la naissance industrielle de la «houille hlanche », c'est-à-dire beaucoup plus tard.

Toujours est-il qu'il a fallu en France attendre l'avènement d'André Coyne pour que la technique des barrages-voûtes reparte sur des idées neuves. Chaque nouvel ouvrage construit marque alors un progrès sur le précédent. Les barrages deviennent plus hauts, plus larges, moins épais et plus élégants. Les détails constructifs se précisent.

Dès 1935, la construction du barrage de Marèges, sur la Dordogne, qui à l'époque constitue d'assez loin un record dans ce domaine, symbolise l'avance acquise par la France dans cette discipline. Pour plusieurs ouvrages, on a déjà dépassé la limite du $c / h$ fixée par Resal et pourtant les apports du calcul sont bien incertains. I a méthode des arcs-plongeants, utilisée croyons-nous pour la première fois à Marćges, constitue certes un progrès sur le calcul pur el simple des arcs horizontaux indépendants, en ce qu'elle prend implicitement en compte le poids propre de l'ouvrage. Mais le mode de découpage du barrage auquel elle conduit est à peine moins arbitraire que précédemment. A Marèges, toutefois, une tendance nouvelle fort intéressante apparaît. La voûte est posée sur un socle, luimême fondé au rocher, et on a réduit l'épaisseur de la voûte dans les zones basses, c'est-à-dire celles situées immédiatement au-dessus du socle, afin de limiter la raideur des consoles, done l'importance des flexions à la base du barrage, en supprimant le béton tendu, donc inutile.
L'encastrement des consoles était, de ce fait, traité effectivement comme une liaison surabondante et parasite. L'idée de l'articulation était née. Nous ne pensons pas que cette idee ait été ultérieurement exploitée comme elle le méritait.

Coyne lui-même pensait que l'hyperstatisme donnait aux ouvrages un suppliment de sécurité qu'il ne fallail pas négliger.

Du point de vue technique, le probleme était et demeure complexe. Par ailleurs, la suppression de l'encastrement au pied ne le résoud que partiellement. En raison de l'ćpaisseur minimale que l'on doit nécessairement donner à la base de la voûte et de l'eftort de compression agissant au niveau du joint, il subsiste généralement un moment de flexion résiduel et un effort tranchant, absorbé par le frottement entre la vonte et son socle.

Néanmoins, l'esprit des projeteurs de barrages s'accommodait progressivement de la présence de ces efforts de flexion au pied de la voûte.

II faut dire que fort peu nombreux sont les barrages qui ont montré de fissurations dans les zones où le calcul approximatif laissail prévoir des contraintes de traction prohibitives. Sans donte doit-on attribuer ce résultat à l'aptitude du béton à la plasticité. A ce point de vue, les barrages sonl placés dans des conditions idéales puisque la zone de béton proche du parement amont est toujours saturéc el que la mise en charge s'effectue d'une manière extrêmement lente et géneralement continue. Mais comme nous l'avons-dit plus hant, il est vraisemblable. que c'est plutôt le rocher qui se "prête", étant toul préparé pour cela. De plus, les fissures de pied amont ne sont observables que lorsque la retenue est vide et les efforts supprimés. Elles ont donc disparu. En charge, elles ne fuient pas, te béton étant comprimé sur le parement aval.

Néanmoins, certains ouvrages se sont nettement fissurés au pier. Il s'agissait souvent d'ouvrages présentant un fort surplomb sur l'aval, circonstance aggravante du point de vue des flexions à la base. Cette fissuration, libérant partiellement la liaison au pied de la voûte, n'a pu que conduire à une meilleure redistribution des efforts, sans compromettre pour atulant l'ćtanchéité puisqu'elle n'intéressait vraisemblablemont pas la totalité de l'épaisseur.

Après Marèges, les barrages-voûtes minces construits en France onl été concus en s'attachant essentiellement ì la définition des ares horizontaux. Chaque are était préalablement étudié séparément de façon que ses caractéristiques soient aussi proches que possible de l'optimum (c'est-à-dire avec un angle au centre tendant vers $130^{\circ}$ ). Les parements amont el aval, respectivement enveloppe de l'extrados et de l'intrados des arcs, résultaient ainsi de la définition des ares et de leur implantation relative, étudjée avec 
le souci de limiter les surplombs sur l'amont défavorables à la stabilité à vide.

Pour séduisant à l'esprit que soit ce processus općratoire, il ne doit pas faire perdre de vue qu'un barrage se comporte avant tout comme une coque, et que le concept des ares horizontaux ne constitue qu'un artifice simpliste de calcul.

Indépendamment de l'influence des conditions au pourtour, des variations trop rapides de courbure et d'épaisseur sont de nature à conduire en réalité à des accroissements locaux des flexions el des torsions dans la voûte. Cela ne peut d'ailleurs apparaitre que par la mise en ouvre d'une. méthode de calcul très poussée, telle que la «Trial Load».

Or, en France, peu de barrages ont fait l'objet d'une vérilication par cette méthode qui, malgré la longueur et la difficulté des calculs auxquels elle conduit, ne peut prétendre en définitive à autre chose qu'à fixer des ordres de grandeur. L'utilisation industrielle des machines électroniques a fort heureusement permis de libérer le projeteur de la partie la plus fastidieuse des calculs.

Longtemps donc, les barrages-voûtes ont été simplement calculés en ares horizontaux indépendants, en supposant en outre rigoureusement indéformable le rocher d'appui. L'introduction des coefficients de Vogt a, par la suite, permis d'apprécier l'influence de la déformation du rocher, dans la mesure bien entendu où la réalité est assez proche des hypothèses du calcul.

Il est à peine besoin de souligner combien il convient d'être prudent dans ce domaine. Les conditions d'isotropie, l'homogénéité et de continuité sur lesquelles est basée la théorie de Boussinesq ne sont jamais remplies en réalité. La surface d'application des efforts sera toujours fort éloignée d'un plan indéfini, et, plus grave, les caractéristiques mécaniques du rocher (module d'élasticité longitudinal et coefficient de Poisson) seront fréquemment variables d'un appui à l'autre, parfois même le long d'un même appui.

Ia prise en comple des déformations $\mathrm{du}$ rocher marque cependant un progrès sur les méthodes précédentes. Pour imprécise qu'elle soit, elle rend compte d'un phénomène réel et apporte des indications nouvelles quant aux conditions au pourtour.

Indépendamment de la «Trial Load», le procédé de vérificalion le plus courant en France, est l'ajustement de clé. Cette méthode, qui rétablit simplement l'égalité des déplacements radiaux entre la console de clé de la voûte et un certain nombre d'ares horizontaux, n'a d'autre ambition que de préciser grossièrement le mode de répartition des charges entre arcs et consoles et n'apporte aucune indication sur les efforts secondaires dans les parties de la voute quelque peu éloignées de la clé.

L'ampleur des calculs qui résultent de l'application de cette méthode cst pourtant loin d'être négligeable.

De ce qui précède, on peut facilement conclure à la nécessité de donner à un barrage-voûte ses formes quasi définitives avant même d'entrer dans le détail des laborieux calculs dont nous venons de parler, c'est-à-dire en n'usant que des « recettes» élémentaires. Si le succès, vis-à-vis des calculs ultéricurs, du résultat ainsi obtenu dépend de l'expérience et de l'intuition du projeteur, il faut bien convenir qu'il dépend aussi de sa chance.

Il est pourtant probable que bien peu de projets ont été remaniés au vu des indications du calcul définitif. Dans un barrage-vôite classique, en effet, quelles que soient par ailleurs les conditions au pourtour et les hypothèses des calculs, les arcs constituent selon toute évidence l'élćment porteur principal de la voûte. C'est pourquoi, en définitive, la prise en compte des flexions et des torsions - qu'il est légitime de regarder comme des phénomènes secondaires n'apportera que des modifications relativement peu importantes aux contraintes dans les ares assez éloignés de la fondation. A cet égard, il ost intéressant de signaler que certains règlements étrangers prescrivent une vérification obligatoire par le calcul des arcs indépendants, quelle que soit par ailleurs la méthode globale adoptée.

Le modèle réduit, où l'on mesure au lien de calculer, se prête évidemmient assez bien aux retouches successives d'un projet. Les indications fournies par ce procédé sont précieuses et complètent utilement celles du calcul. Elles peuvent néanmoins être également assez loin de la réalité en raison notamment de l'impossibilité de reproduire les conditions au pourtour et des difficultés à respecter strictement les lois de similitude (en particulier en ce qui concerne le poids, facteur pourtant non négligeable dans de nombreux cas).

Alors qu'en France, les ingénieurs semblaient se stabiliser sur la doctrine des arcs horizontaux et de l'ajustement de clé, les constructeurs étrangers, surtout des Suisses et les Italiens s'attachaient à la conception en coque des barrages-voûtes, et édifiaient de remarquables ouvrages à double courbure.

La définition des consoles n'était plus, dès lors, le fait exclusif des caractéristiques des ares et de leur implantation relative. L'étude particulière des consoles visait à utiliser les possibilités de la deuxième courbure, non seulement pour soulager les ares horizontaux, mais aussi et surtout pour réduire l'importance des flexions le long des consoles et notamment à leur base. 
De même les modèles réduits cherchaient et réussissaient à prendre en compte le poids propre.

Parallèlement à cette évolution, les ingénieurs italiens imaginaient le joint périmétral qui, à notre avis, présente de sérieux avantages :

$1^{\circ}$ Il permet une régularisation géométrique du contour de la voûte. En effet, Jorsque l'on fonde directement la voûte sur le rocher d'appui, on lui fait épouser la configuration du rocher sain, laquelle est souvent fort irrégulière. Le joint périmétral sépare nettement la fonction voûte de la fonction appui. L'appui est constitué par un socle continu épais qui joue un rôle d'intermédiaire entre la voûte et sa fondation. Il est donc possible de compenser les irrégularités de Ja roche en jouant sur l'épaisseur du socle. La lisière de la voûte se présente alors suivant une ligne continue et régulière et l'on ne peut nier l'intérêt de cette disposition vis-à-vis du phénomène de concentration locale des contraintes;

$2^{\circ}$ Il conduit à une meilleure répartition des contraintes sur le rocher en amorçant la diffusion des poussées qu'il reçoit;

$3^{\circ}$ Il crée le long du pourtour de la vonte une liaison unilatérale susceptible de localiser les tendances éventuelles à fissuration.

On peut cependant se demander comment doit être considéré le joint périmétral en regard des concepts classiques de la résistance des matériaux. La section représentée par ce joint peut subir une légère rotation due aux déformations élastiques de la vô̂te et du socle. Ce n'est donc ni un encastrement, dans lequel par definition la section considérée ne peut tourner, ni une articulation, où la fibre neutre tourne librement. Bien que, théoriquement, il soit possible d'ćtablir les équations liant en tout point du joint la rotation aux efrorts transmis si l'on connail leur excentricité, les calculs deviennent singulièrement compliqués.

Aussi est-on amené à penser que le joint périmétral cst encore une solution incomplete, et que c'est vers l'articulation que l'on doit s'orienter pour utiliser à fond la technique des barrages-routtes à double courbure.

En résumé, nous pensons que la conception des barrages-voûtes minces devrait conduire à : $1^{\circ}$ des ouvrages à double courbure plus accentuée s'appuyant

$2^{\circ}$ sur un élément du type «pulvino», permettant de ne transmettre au rocher que des contraintes faibles et, dans la mesure du possible, normales aux surfaces de contact, par l'intermédiaire

$3^{\circ}$ d'une articulation supprimant les efforts parasites dûs à l'encastrement.

Dans un prochain article, nous examinerons le calcul des ouvrages minces par la thérie de la membrane. Nous verrons ensuite comment il parait aujourd'hui possible de faire coïncider la réalité et le calcul, en articulant les ouvrages minces, et comment on peut en môme lemps s'afrranchir en grande parlie des imperfections du rocher.

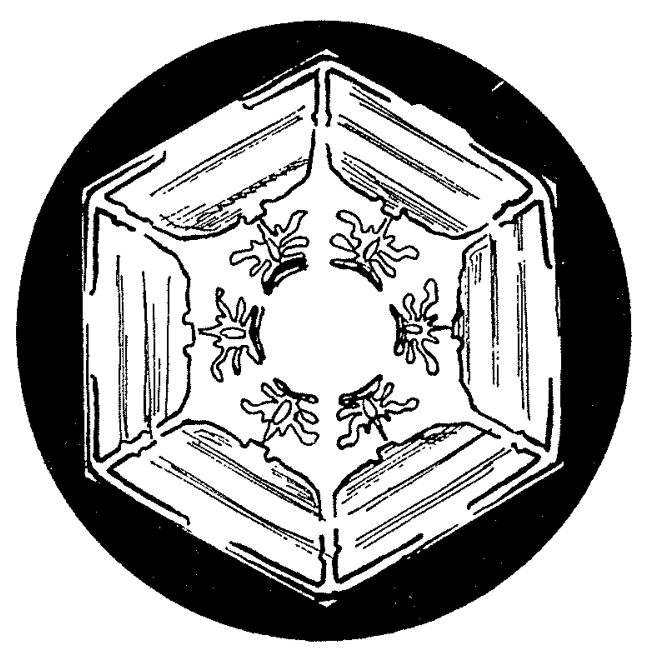

